2005

\title{
On-axis parallel ion speeds near mechanical and magnetic apertures in a helicon plasma device
}

Xuan Sun

\author{
S. A. Cohen
}

Earl E. Scime

Mahmood Miah

Follow this and additional works at: https://researchrepository.wvu.edu/faculty_publications

\section{Digital Commons Citation}

Sun, Xuan; Cohen, S. A.; Scime, Earl E.; and Miah, Mahmood, "On-axis parallel ion speeds near mechanical and magnetic apertures in a helicon plasma device" (2005). Faculty Scholarship. 268.

https://researchrepository.wvu.edu/faculty_publications/268 


\section{On-axis parallel ion speeds near mechanical and magnetic apertures in a helicon plasma device}

Xuan Sun, S. A. Cohen, Earl E. Scime, and Mahmood Miah

Citation: Physics of Plasmas 12, 103509 (2005); doi: 10.1063/1.2121347

View online: https://doi.org/10.1063/1.2121347

View Table of Contents: http://aip.scitation.org/toc/php/12/10

Published by the American Institute of Physics

\section{Articles you may be interested in}

Ion acceleration in plasmas emerging from a helicon-heated magnetic-mirror device

Physics of Plasmas 10, 2593 (2003); 10.1063/1.1568342

Current-free double-layer formation in a high-density helicon discharge

Applied Physics Letters 82, 1356 (2003); 10.1063/1.1557319

High temperature electrons exhausted from rf plasma sources along a magnetic nozzle

Physics of Plasmas 24, 084503 (2017); 10.1063/1.4990110

The ion velocity distribution function in a current-free double layer

Physics of Plasmas 12, 093502 (2005); 10.1063/1.2033647

Laboratory evidence of a supersonic ion beam generated by a current-free "helicon" double-layer

Physics of Plasmas 11, 1706 (2004); 10.1063/1.1652058

On plasma detachment in propulsive magnetic nozzles

Physics of Plasmas 18, 053504 (2011); 10.1063/1.3589268

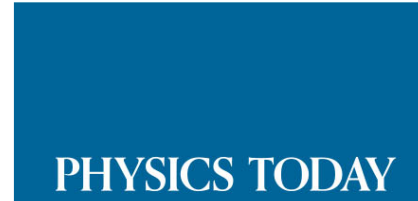

MANAGER'S GUIDE

WHITEPAPERS

\section{READ NOW}

PRESENTED BY

Accelerate R\&D with

Multiphysics Simulation 


\title{
On-axis parallel ion speeds near mechanical and magnetic apertures in a helicon plasma device
}

\author{
Xuan Sun \\ Department of Physics, West Virginia University, Morgantown, West Virginia 26506 \\ S. A. Cohen \\ Princeton Plasma Physics Laboratory, Princeton, New Jersey 08543 \\ Earl E. Scime \\ Department of Physics, West Virginia University, Morgantown, West Virginia 26506 \\ Mahmood Miah \\ Princeton Plasma Physics Laboratory, Princeton, New Jersey 08543
}

(Received 9 August 2005; accepted 22 September 2005; published online 27 October 2005)

\begin{abstract}
Using laser-induced fluorescence, measurements of parallel ion velocities were made along the axis of a helicon-generated Ar plasma column whose radius was modified by spatially separated mechanical and magnetic apertures. Ion acceleration to supersonic speeds was observed $0.1-5 \mathrm{~cm}$ downstream of both aperture types, simultaneously generating two steady-state double layers (DLs) when both apertures were in place. The DL downstream of the mechanical aperture plate had a larger potential drop, $\Delta \phi_{\mathrm{DL}}=6-9 k T_{e}$, compared to the DL downstream of the magnetic aperture, $\Delta \phi_{\mathrm{DL}} \sim 3 k T_{e}$. In the presheath region upstream of the mechanical aperture, the convective ion speed increased over a collisional distance; from stagnant at $4 \mathrm{~cm}$ from the aperture to the 1.4 times the sound speed at the aperture. The dependence of the free- and trapped-ion-velocity-distribution functions on the magnetic-field strength and mechanical-aperture electrical bias are also presented. () 2005 American Institute of Physics. [DOI: 10.1063/1.2121347]
\end{abstract}

\section{INTRODUCTION}

Electrostatic double layers (DLs) in plasmas are isolated non-quasi-neutral regions remote from material surfaces. ${ }^{1}$ The measured electric potential drops across the DLs, $\Delta \phi_{\mathrm{DL}}$, range from 3 to $30 k T_{e} / e$ and the DL length, typically 30-100 Debye lengths, $\lambda_{D}$, is much shorter than the electron-electron or ion-neutral lengths. The resulting strong electric field in the collisionless plasma creates energetic particle beams. Usually, ${ }^{2,3}$ the ion-velocity-distribution function (IVDF) on the low-potential side of the DL contains the energetic beam and a low-energy ion population. The lowenergy ion population on the low-potential side of the DL consists of a single Maxwellian distribution symmetric around the zero speed and is often called the "trapped" population in the literature. Ions, which originate on the highpotential side of the DL, accelerate through the DL, and form the energetic beam on the low-potential side, are termed "free." (Similar labels are applied to electrons on the highpotential side.) The formation of DLs in laboratory plasmas may be promoted by a number of different means. The methods we employ use mechanical and magnetic apertures, both individually and in tandem.

A DL is frequently identified by measuring the spatial change of the plasma potential with Langmuir probes. Extracting, from probe data, the convective speed of ions or electrons transiting through or reflected by the DL is a difficult, and sometimes impossible, task. Retarding-field energy analyzers (RFEAs) have been used with success to measure the free- and trapped-electron and ion energy distributions. Laser-induced fluorescence (LIF) is a nonperturbing method to determine the velocity distribution of ions and atoms. There have been numerous LIF studies of ion flows in helicon plasma experiments. ${ }^{4-6}$ The results presented here are the first LIF measurements in helicon sources to provide detailed IVDFs upstream, in, and downstream of a DL for both trapped and free ions and with multiple DLs generated by tandem apertures.

Researchers using helicon plasma sources have reported the formation of current-free DLs at distances many helicon wavelengths from the helicon antennas. With Ar helicon plasmas generated at low pressure, $<0.5 \mathrm{mT}$, Charles and Boswell ${ }^{7}$ used a RFEA to study a DL with $\Delta \phi_{\mathrm{DL}}=3 k T_{e} / e$ formed at the junction of their Pyrex helicon source with a metal expansion vessel. Both the potential profile and the energy of the ion beam accelerated through the DL were determined. ${ }^{8}$ In the same source, but for hydrogen plasmas, Charles ${ }^{9}$ also observed a supersonic ion beam, 2.1 $C_{s}$ (where $C_{S}=\sqrt{k T_{e} / M_{i}}$ and is the ion sound speed), and attributed the beam to the presence of a double layer. Using a tunable diode-laser-based laser-induced-fluorescence diagnostic, ${ }^{10}$ Cohen et al. ${ }^{6}$ reported supersonic velocities of argon ions which had passed through a mechanical aperture located $1 \mathrm{~cm}$ upstream of a region of converging-diverging magnetic field, termed a magnetic nozzle or a magnetic aperture. Supersonic flows were only detected under the conditions of high-helicon axial-power flow $\left(>30 \mathrm{~W} / \mathrm{cm}^{2}\right)$ and lowambient-gas pressure $(<1.5 \mathrm{mT})$ in the helicon source. In the center of the helicon source chamber, about $30 \mathrm{~cm}$ upstream of an aperture plate and $30 \mathrm{~cm}$ downstream of the helicon antenna, ions were stagnant, i.e., their flow speed was below 


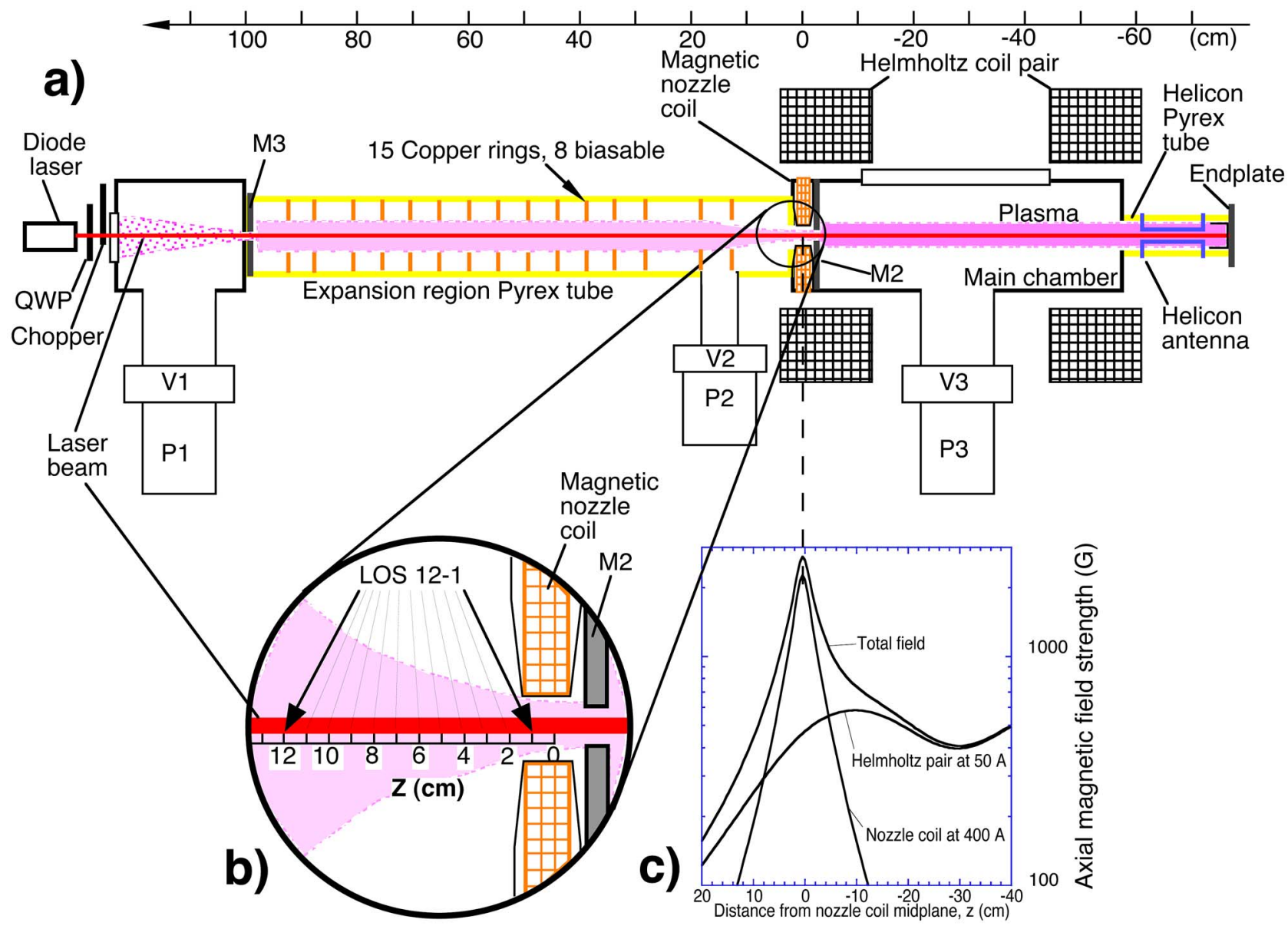

FIG. 1. (Color online). (a) The schematic of the Magnetic Nozzle eXperiment (MNX). Argon plasma is formed by the absorption of helicon waves launched from a double-saddle antenna. The plasma flows through the main chamber along the magnetic-field lines created by a set of Helmholtz coils. The plasma then flows through a metal aperture M2 and the nozzle coil into the expansion region (ER). The beam of a diode laser is directed along the MNX axis, allowing LIF measurements throughout the MNX. (b) The scanning mechanism for the LIF collection optics allows 12 lines-of-sight (LOS) intercepting axial points in the ER near the nozzle. (c) The axial field strength near the nozzle at a Helmholtz-coil current of $50 \mathrm{~A}$ and a nozzle current of $400 \mathrm{~A}$, typical of experimental conditions in this paper.

the ion thermal speed. The rapid acceleration of ions as they passed through the aperture was the evidence that a DL existed in the nozzle region. Limited optical viewing access did not permit LIF measurements near the magnetic-nozzle midplane. Also using a LIF system, Sun et al. ${ }^{5}$ reported that at $\sim 1 \mathrm{~m}$ from the antenna in a low-axial-power flow $\left(\sim 5 \mathrm{~W} / \mathrm{cm}^{2}\right)$, large-radius, low-neutral-pressure helicon argon plasma source, a supersonic ion beam developed where the plasma exited the source chamber into an expansion chamber. In addition to the ion beam, a cold, subsonically drifting background ion population was observed.

Features common to these and several earlier nonhelicon DL-producing experiments are apertures-mechanical or magnetic-and low-neutral-gas pressures. In the context of the physics of double layers, what is an aperture? An aperture (in a plate) is a hole of radius, $r_{a}$, smaller than the plasma column radius, $r_{p}$, which divides the plasma column into source and expansion regions, see Fig. 1. Mechanicalaperture plates establish an equipotential boundary in a plane around the aperture hole and on the surfaces surrounding the hole. In the experiments described here, the short sheath thickness, ${ }_{s} \lambda_{D}<10^{-3} \mathrm{~cm}$, in the source chamber results in a strong electric field at the aperture plate's surface, $>10^{4} \mathrm{~V} / \mathrm{cm}$. If the sheath thickness were to exceed the aperture diameter, ions would fall through the sheath and pass through the aperture with the same velocities they would achieve in the absence of the aperture, i.e., the plasma does not "see" the aperture. ${ }^{11}$ Since the presheath has a characteristic length $\sim 3 \mathrm{~cm}$, which is much larger than the aperture diameter, a similar effect might be expected for ions passing through the presheath. In other words, the ions might be accelerated to the aperture by presheath electric field.

A magnetic aperture is a region of converging/diverging magnetic field, and was originally called a magnetic Laval nozzle. Supersonic ion beams, with Mach numbers as large as 3, were obtained using such nozzles as long ago as $1969 .{ }^{12}$ More recently, a magnetic nozzle has been proposed for the VASIMR rocket to convert thermal energy into thrust. ${ }^{13,14}$ Note that, in the measurements reported here and earlier, the ion-beam energy decreased with increasing nozzle field strength. Therefore, the ion acceleration to supersonic speeds is not simply understood by analogy to the mechanical Laval nozzle. Efforts must be made to understand the static electric field, i.e., the DL, which creates the energetic ion beam. 
Even for a purely divergent magnetic-field configuration (no compression region), Sun et al. observed supersonic ion flow speed that increased with decreasing magnetic-field strength in the expansion region. ${ }^{5}$

Static electric double layers can form in a current-free plasma expanding in a divergent magnetic field was predicted in an analytical study by Perkins and Sun in $1981 .{ }^{15}$ Although they predicted that a stable DL required $T_{e}<T_{i}$, there is strong experimental evidence that stable DLs can exist in expanding plasmas with $T_{e}>T_{i}$ in the absence ${ }^{7}$ or presence ${ }^{16}$ of current. More recently, a one-dimensional, hybrid simulation (particle ions and fluid electrons) that modeled rapid plasma expansion in a diverging magnetic field with an axial-position-dependent electron-loss rate in a uniform magnetic field showed that the rapid decrease in plasma density, such as due to a diverging magnetic field, is consistent with the double-layer formation in a current-free plasma. ${ }^{17}$ In that simulation, a thin $\left(\sim 7 \lambda_{D}\right) \quad 14 \mathrm{eV} \mathrm{DL}$ formed at the location of rapid plasma expansion for upstream parameters of $0.5 \mathrm{mTorr}, n_{e}=6.5 \times 10^{8} \mathrm{~cm}^{-3}$, and $T_{e}$ $=7.2 \mathrm{eV}$. Throughout the simulation volume, a low-energy population of ions, corresponding to ions created by ionization and by charge-exchange collisions, was observed. Downstream of the DL, a high-energy ion population, corresponding to ions accelerated through the DL potential drop, was observed in addition to the low-energy background population. The total ion acceleration, i.e., presheath and DL, occurred over roughly an ion mean free path and the presheath thickness, or length, was much longer than the length of the DL.

A recent experiment by Plihon et al. confirmed DL formation in an axially uniform plasma with a uniform magnetic field and a strong axial density gradient. ${ }^{18}$ By puffing $\mathrm{SF}_{6}$ gas into the plasma at a single axial location, the highly electronegative $\mathrm{SF}_{6}$ gas created a strong electron-density gradient along the plasma axis by substantially reducing the electron density - thereby simulating rapid plasma expansion without a divergent magnetic field.

To the best of our knowledge, no computational study of the effects of overall magnetic-field strength on the DLs is available in the literature. As will be shown by these experiments, there is a clear correlation among the strength of the magnetic field, the magnitude of the potential drop across the DL, and the floating potential of an aperture placed in the plasma. The large floating potentials of the aperture are suggestive of the presence of energetic electrons in the helicon plasma. Though suprathermal electrons are expected in helicon plasma devices, because of the high plasma densities of helicon plasmas, experimental verification of their existence and properties is a hotly debated issue in the helicon community. Since a small population, $\sim 10 \%$, of energetic electrons in a cooler background plasma has been shown to induce the formation of a freestanding current-free double layer, ${ }^{19}$ the DL measurements presented in this work may also shed light on the question of energetic electrons in helicon plasmas. Other experiments demonstrated that smaller energetic populations, as low as $1 \%$ of the bulk, are sufficient to sustain a DL and that the DL strength increased with the number of energetic electrons. ${ }^{20}$
There are also other questions concerning the role of an aperture in a plasma. For example, will the presheath electric field be affected if an aperture is created in an absorbing wall? That the length of presheath should be roughly equal to the ion-neutral collision length is well known and was employed in Riemann's model of plasma sheaths, ${ }^{21}$ which was verified experimentally by Oksuz and Hershkowitz on a surface immersed in a low-density, low-temperature, weakly collisional, argon plasma. ${ }^{22}$ They found that the potential drop across the presheath is $\Delta \phi_{\mathrm{ps}} \sim k T_{e} / e$, instead of $k T_{e} / 2 e$ as is common at a boundary between the sheath and presheath. The experimental data that will be presented in this work confirm that the potential drop is $\sim k T_{e} / e$ in front of an aperture in a metal plate. The role of a small superthermal electron population in determining $\Delta \phi_{\mathrm{ps}}$ as well as $\Delta \phi_{\mathrm{DL}}$ cannot be discounted. ${ }^{23} \mathrm{We}$ also have installed two apertures, separated by up to $10^{5} \lambda_{D}$, to explore whether the strongly modified (upstream) IVDF and electron energy distribution function (EEDF) will promote formation of a second DL at the second aperture.

With a tunable diode-laser-based LIF diagnostic, we have mapped the argon IVDF on the high- and low-potential sides of a DL localized beyond an aperture in a metal plate located many wavelengths from the helicon source. The strength and spatial extent of the DL were determined from the measurements of the spatial dependence of the free-ion energy downstream of the DL. The effect of the location of the aperture plate-relative to the magnetic-field coils-on the DL was investigated by repositioning the aperture plate to the following locations: (1) the center of the main chamber, (2) the magnetic-nozzle region at the end of the main chamber, and (3) the expansion region downstream of the magnetic-nozzle coil. Without any aperture plate, the IVDF measurements show that a $20 \mathrm{~V}$ potential drop develops over a distance of $3 \mathrm{~cm}, \sim 50{ }_{s} \lambda_{D}$, beyond the 2-cm-i.d. magnetic nozzle at the end of the main chamber. (The nozzle coil acts as both a mechanical and a magnetic aperture.) With a mechanical-aperture plate also placed in the plasma, an additional DL forms. Short regions, $\sim 0.5 \mathrm{~cm}$, of free-ion deceleration in the DL and also backflowing (reflected or trapped) cold ions have been observed, suggesting that the DL is of the ion-acoustic type. Ion acceleration in the presheath was also measured by LIF.

\section{EXPERIMENTAL APPARATUS}

The experiments were performed in the Magnetic Nozzle Experiment (MNX) Facility (see Fig. 1). A 4-cm-diam, steady-state helicon plasma flows along the magnetic field formed by a Helmholtz-coil pair. The plasma exits the source (or main) chamber through a coaxial 2-cm-i.d., 3 -cm-long nozzle coil used to control the magnetic-field gradient. The nozzle coil extends from $z=-1.5 \mathrm{~cm}$ to $z$ $=1.5 \mathrm{~cm}$. Figure 1(b) shows the axial field strength near the nozzle at a Helmholtz-coil current of $50 \mathrm{~A}$ and nozzle current of $400 \mathrm{~A}$, typical of experimental conditions in this paper. Exiting the nozzle coil, the plasma enters a 10-cm-i.d., 100-cm-long Pyrex tube termed the expansion region (ER). The ER has 15 internal 4-cm-i.d. coaxial copper rings, of 
which eight may be electrically biased. The floating potentials of the copper rings in the ER were typically -40 to $-120 \mathrm{~V}$. Such large floating potentials suggest the presence of energetic electrons in the ER. Floating potentials in the main chamber are much lower, $\sim-30 \mathrm{~V}$, indicating that an energetic electron population, if present, has a population less than $\left(m_{e} / M_{\mathrm{Ar}}\right)^{1 / 2} \sim 1 / 300$ smaller than that of the bulk electrons. ${ }^{24,25}$ Briefly, the floating potential in a two-electrontemperature plasma only moves away from the value that would exist with only the primary (cold) component present, when the ion flow to objects in the plasma is balanced by the hot-electron component alone.

Also shown in Fig. 1(a) are three electrically biasable metal disks, labeled end plate, M2, and M3. For the experiments reported here, the end plate and M3 were electrically floating. The disk M2, i.e., the aperture plate, has a hole, the aperture, which limits the plasma and neutral-gas flows and helicon-wave propagation into the ER. Figure 2 shows the five locations where M2 may be positioned. Also, M2 may be completely removed, as shown in Fig. 2(a). (The aperture diameters and plate thicknesses are indicated on the figure.) Sheaths of differing thickness will form on the opposite sides of the aperture plate, predominantly because of the different plasma densities on the two sides of the plate. Based on Langmuir probe measurements at the center of the main chamber and in the expansion chamber $10 \mathrm{~cm}$ from the aperture, the ratio of the Debye lengths in the ER to that in the source chamber $\left({ }_{\mathrm{ER}} \lambda_{D} /{ }_{s} \lambda_{D}\right)$ is $\sim 10$ with ${ }_{s} \lambda_{D} \sim 6 \times 10^{-4} \mathrm{~cm}$. The control of pumping speed in the ER allows the ratio of ion-neutral collision lengths to be varied, $0.1<_{\mathrm{ER}} \lambda_{\text {in }} /{ }_{s} \lambda_{\text {in }}$ $<10$, with $1<{ }_{s} \lambda_{\text {in }}<10 \mathrm{~cm}$.

For LIF measurements, the laser is directed down the axis of the plasma column, through the entire ER and main chamber into the helicon antenna region and onto the end plate. Before entering the MNX vacuum chamber, the laser is sent through a quarter-wave plate, allowing creation of either right or left circularly polarized light for exciting either the $\sigma-$ or $\sigma+$ transitions in $\mathrm{Ar}^{*+}$. The shift in the center wavelength of the measured LIF signal is used to determine the average flow of the ions along the laser path. A detailed description of the LIF measurement principles can be found in Ref. 11.

\section{EXPERIMENTAL RESULTS AND DISCUSSION}

\section{A. The magnetic nozzle as an aperture}

Without an aperture plate, Fig. 2(a), the plasma flows into the expansion region from the main chamber through the 2-cm-i.d. magnetic-nozzle coil. The midplane of the nozzle coil is defined as $z=0 \mathrm{~cm}$. Figure 3 shows the flow speed at $z=3.0 \mathrm{~cm}$ in the ER versus the nozzle magnetic-field strength for a rf power of $800 \mathrm{~W}$, magnetic field $\left(B_{H}\right)$ of $580 \mathrm{G}$ at the center of the source chamber, and neutral pressures of 0.7 and 0.2 mTorr in the source $\left(P_{M}\right)$ chamber and ER $\left(P_{\mathrm{ER}}\right)$, respectively. The energy of the exiting ion beam decreases with increasing nozzle field strength until the (added) nozzle field strength reaches $2000 \mathrm{G}$. (At $B_{n}=2000 \mathrm{G}$, the ratio, $R$, between the on-axis magnetic field at the nozzle midplane to that in the center of the ER was
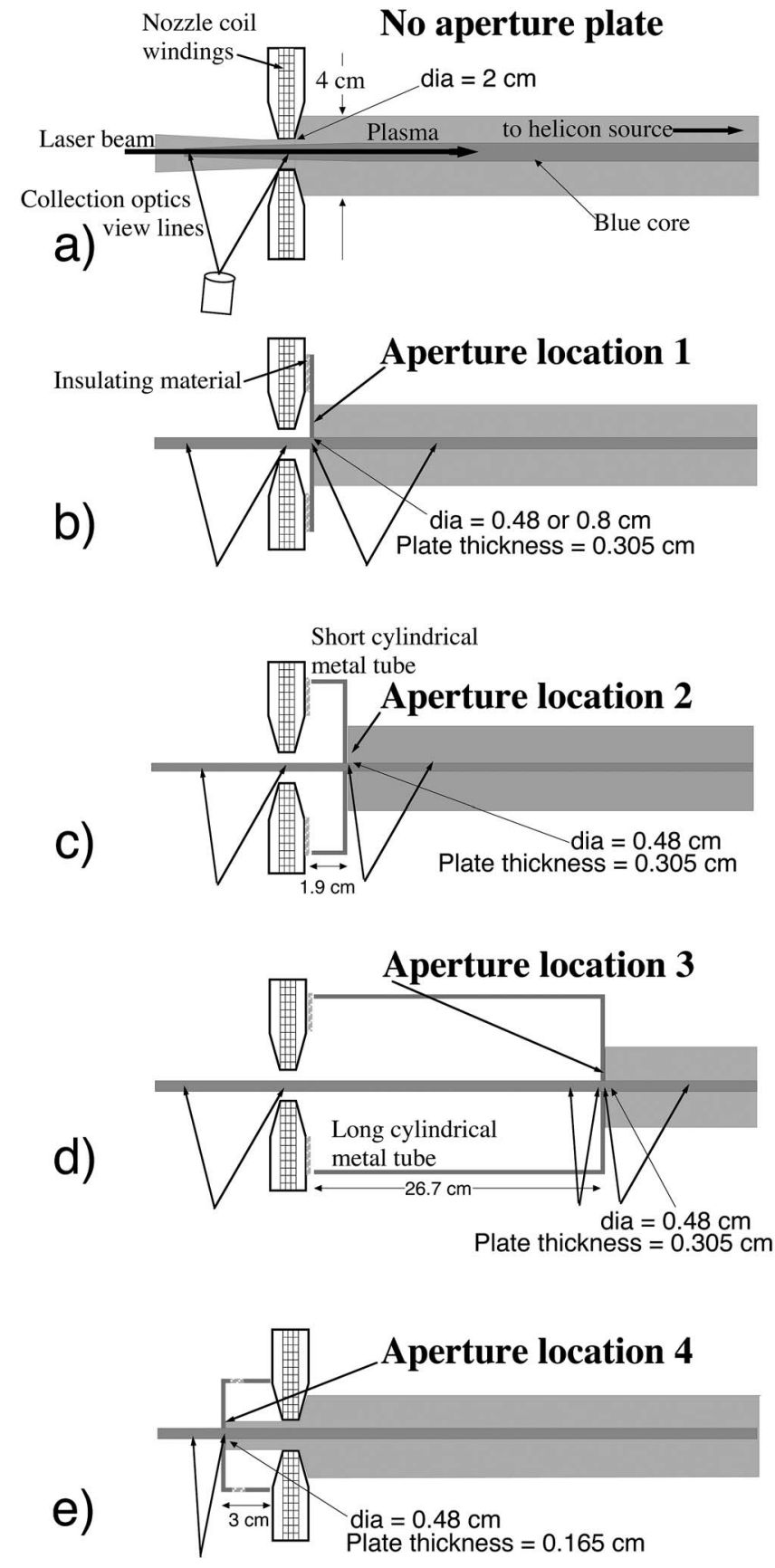

FIG. 2. Five different configurations were used: (a) The experiments without an aperture plate and the measurement were performed in the ER. (b) A metal disk with an aperture of $0.48 \mathrm{~cm}$ and thickness of $0.305 \mathrm{~cm}$ was placed immediately before the nozzle. The measurements were performed in both the ER and source (measurements performed with the $0.8 \mathrm{~cm}$ aperture are not presented here). (c) The aperture used in (b) was moved $1.9 \mathrm{~cm}$ into source (data for this configuration are not reported in this paper). (d) The aperture used in (b) was moved $27.6 \mathrm{~cm}$ into the source and measurements were performed near the aperture plate and in the ER. (e) A metal disk with an aperture of $0.48 \mathrm{~cm}$ and thickness of $0.165 \mathrm{~cm}$ was placed in the ER. The measurements were performed near the aperture plate.

$R=4.75$. At $R=4$, a 4 -cm-diam plasma column will pass through the nozzle without contacting the nozzle coil housing.) The ion-beam energy at $z=3.0 \mathrm{~cm}$ is approximately $7 \mathrm{eV}$ for $B_{N}=2000 \mathrm{G}$. The corresponding Mach number $\left(V / C_{s}\right)$ was $\sim 1.3$. For nozzle magnetic-field strengths below $1000 \mathrm{G}$, the LIF signal was too weak to give a good measure of ion speed. 


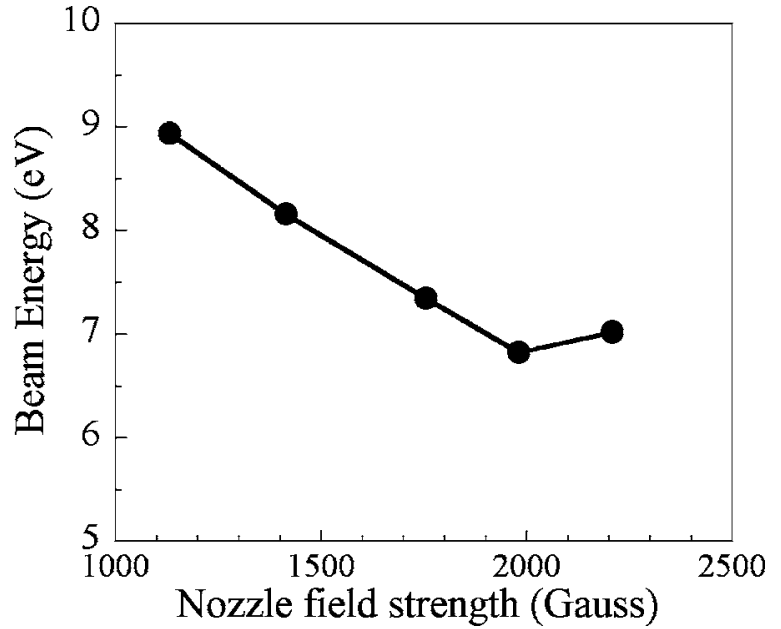

FIG. 3. The beam energy vs the nozzle field strength at $z=3.0 \mathrm{~cm}$ for $\mathrm{rf}$ power of $800 \mathrm{~W}, B_{H}=580 \mathrm{G}, P_{M}=0.7 \mathrm{mTorr}, P_{\mathrm{ER}}=0.2 \mathrm{mTorr}$, and no aperture plate (M2).

Our earlier published data at higher $B_{H}$ fields of $1200 \mathrm{G}$ and with a mechanical aperture located in front of the magnetic-nozzle coil, as in Fig. 2(b), Ref. 6, showed qualitatively similar behavior, i.e., a $5 \%$ decrease in ion energy, $E_{i}$, with increasing $B_{n}$, for $0<B_{n}<2000 \mathrm{G}$, but $E_{i}$ rising $3 \%$ for $2000 \mathrm{G}<B_{n}<3000 \mathrm{G}$. Those earlier results showed considerably higher flow energies $\left(E_{i} \sim 18 \mathrm{eV}\right)$ and speeds, $M \sim 1.7$ at $z=2 \mathrm{~cm}$. At these lower $B_{H}$ values, $R<4$ at $B_{n}=2000 \mathrm{G}$, the effect of the nozzle magnetic field is qualitatively similar to that of a purely magnetic aperture.

\section{B. Mechanical aperture plate}

By placing the aperture plate (AP) at four different positions relative to the midplane of the magnetic-nozzle coil, we investigated the effect of aperture-plate location on the parallel ion flow speed. A $0.1 \mathrm{eV}$ argon ion would have a gyroradius of $0.1-1 \mathrm{~cm}(5000-500 \mathrm{G})$, comparable to the radii of the various apertures used, $0.25-0.4 \mathrm{~cm}$. The transit time for ions, accelerated by the presheath to $5 \mathrm{eV}$, to pass through the thin aperture plate is 2-20 times shorter than the ion gyroperiod. Independent of the aperture-plate installation, the ion flow speed (energy) in the center of the main chamber is very small, less than $0.03 \mathrm{eV}$. The perpendicular ion temperature is slightly higher, $\sim 0.05-0.5 \mathrm{eV}$. Thus, ions pass through the aperture on nearly straight lines, within $30^{\circ}$ of the plate normal.

\section{Aperture plate immediately upstream of nozzle coil}

With the AP positioned, as shown in Fig. 2(b), just upstream of the AP, at $z=-2.3 \mathrm{~cm}$, the ion flow energy increases to $1.1 \mathrm{eV}$ (Fig. 4). After the $\mathrm{AP}$ and nozzle region, the ion flow energy increases further to $13.0 \mathrm{eV}$ at $z=2.4 \mathrm{~cm}$. By $z=7.4 \mathrm{~cm}$, the ion-beam energy is up to $17.7 \mathrm{eV}$. Coexistent with the ion beam is a low-energy population in the ER. Throughout this paper we use the terminology introduced in Ref. 6: high-energy particles are called HEPs, and low-energy particles are called LEPs. The LEPs, represented by the diamond symbols in Fig. 4, have zero net

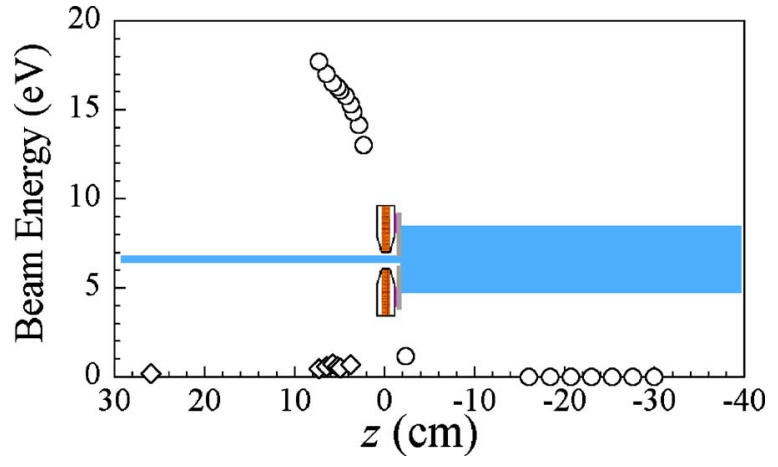

FIG. 4. The beam energy vs $z$ for AP at $z=-1.8 \mathrm{~cm}$ and plasma conditions of rf power $P=600-900 \mathrm{~W}, B_{H}=580 \mathrm{G}, B_{N}=2250 \mathrm{G}, P_{M}=0.6 \mathrm{mTorr}$, and $P_{\mathrm{ER}}=0.3-0.7 \mathrm{mTorr}$. The open diamonds label the ions created locally in the EP (the LEP). The open circles label the ions in and emanating from the source (which become HEP ions in the ER).

flow throughout the expansion region. The lack of LEP net flow persists even in the DL where the HEP ions accelerate from $7800 \mathrm{~m} / \mathrm{s}(12.7 \mathrm{eV})$ to $9200 \mathrm{~m} / \mathrm{s}(17.6 \mathrm{eV})$ in $4.6 \mathrm{~cm}$. These observations are consistent with the LIF-measured IVDFs in other helicon DL experiments. ${ }^{26}$

Although the LEP ion peak was generally stagnant (as shown later in Fig. 7) or had slightly positive speeds (as shown in Fig. 4), significant LEP ion flows in the $-z$ direction were observed with higher rf power $(950 \mathrm{~W})$ and lower neutral pressure (0.4 mTorr), as shown in Fig. 5. Locations distant from the nozzle, e.g., at $z=8.7 \mathrm{~cm}$, showed a single Maxwellian with no axial flow. Locations closer to the nozzle showed an increasing flow back towards the nozzle, which suggests that a modest negative potential dip occurs downstream of the DL. The depth of the potential dip is at least $0.2 \mathrm{~V}$, or about a hundredth of the DL potential drop. Thus the potential does not decrease monotonically as there is a slight increase in the potential on the downstream side of the DL. Such potential structures are characteristic of DLs that evolve from ion-acoustic solitons. ${ }^{1,27}$

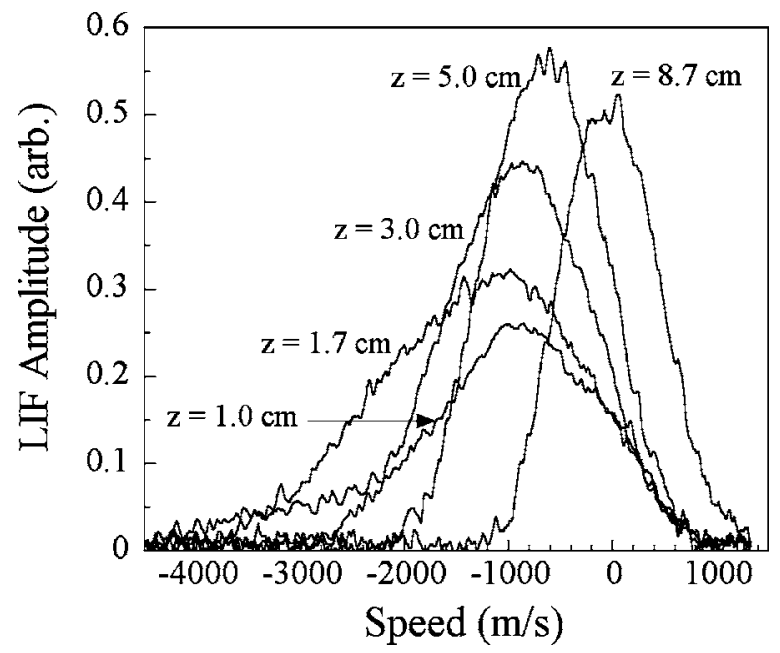

FIG. 5. Example of the reversed flow of LEP ion population at various axial positions. The negative speeds indicate the ions moving upstream towards the source. 


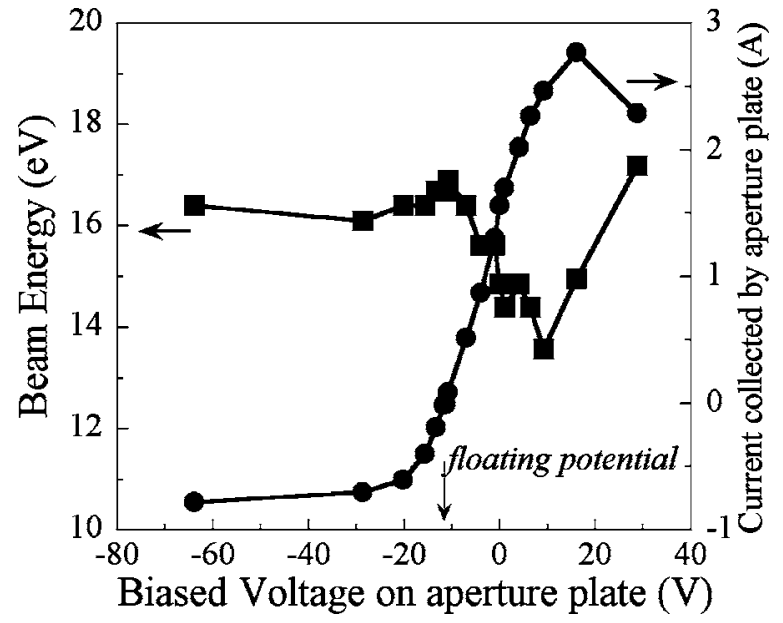

FIG. 6. The ion-beam energy vs the bias voltage on the aperture plate at $z=-3.2 \mathrm{~cm}$ for rf power of $700-800 \mathrm{~W}, B_{H}=580 \mathrm{G}, B_{N}=1700 \mathrm{G}, P_{M}$ $=0.7 \mathrm{mTorr}$, and $P_{\mathrm{ER}}=0.3 \mathrm{mTorr}$. The aperture plate was at $z=-1.8 \mathrm{~cm}$.

Figure 6 shows the ion-beam energy measured at $z=3.2 \mathrm{~cm}$ and the current collected by the aperture plate versus a bias voltage applied to the AP. The minimum ion-beam energy occurs at a bias voltage of $9.1 \mathrm{~V}$ (close to the measured plasma potential of $9.8 \pm 1.0 \mathrm{~V})$. When the AP is biased more negative than the plasma potential, the ion-beam energy increases until the bias voltage equals the floating potential. Further decreases in the applied bias potential lower the ion-beam energy slightly. At the negative potentials, -30 to $-70 \mathrm{~V}$, the AP only collects ion saturation current as the electron current is negligible. A bias voltage above the plasma potential, from 10 to $30 \mathrm{~V}$, also increases the ionbeam energy. Electron saturation current to the AP is not achievable with the current and voltage capabilities of the AP biasing power supply and instability of the plasma column. It is important to note that the DL may no longer be a currentfree DL when the AP is biased.

For an expanding, two-electron-temperature plasma terminated with a metal plate at one end, Hairapetian and Stenzel reported that the DL amplitude decreased as an increasing positive bias voltage was applied to the end plate. $^{20,28}$ They reported that the DL disappeared at large positive bias voltage and that negative bias voltages had no effect on their DL. Consistent with their results, a large negative bias voltage had little effect on the ion-beam energy in these experiments. However, the detailed LIF measurements indicate that the ion-beam energy does decrease slightly with negative bias until the AP enters ion saturation-suggesting a slight weakening of the DL until the maximum ion current is pulled through the sheath onto the AP. Similarly, and consistent with the Hairapetian and Stenzel observations, the ionbeam energy also decreases with increasing positive AP bias voltage until the bias voltage equals to $9.1 \mathrm{~V}$ or close to the plasma potential $(9.8 \mathrm{~V})$. We hypothesize that increasing the electron current into the DL (through the positive bias voltage) increases the ratio of thermal to energetic electron densities - thereby decreasing the strength of the DL. ${ }^{20,28}$ In contrast to the Hairapetian and Stenzel results, at large positive bias voltages (when the AP enters into electron satura-

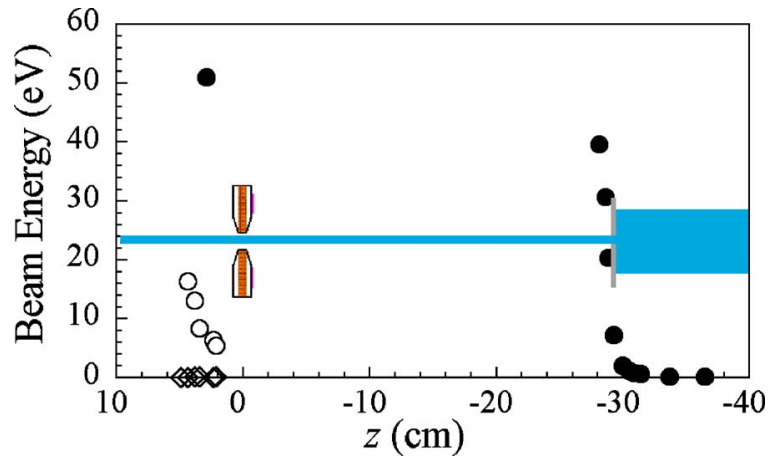

FIG. 7. $\mathrm{AP}$ at $z=-29.4 \mathrm{~cm}$ and plasma conditions of $P=800 \mathrm{~W}, B_{M}$ $=580 \mathrm{G}, B_{N}=1100 \mathrm{G}, P_{M}=0.51 \mathrm{mTorr}$, and $P_{\mathrm{ER}}=0.11 \mathrm{mTorr}$. For measurements in the main chamber, the nozzle magnetic-field strength was decreased to $200 \mathrm{G}$. The open diamonds, open circles, and solid circles denote the parallel kinetic energy of LEP, HEP, and SHEP. The (super-high-energypopulation) (SHEP) label identifies the ions in the nozzle region that are stationary in source and believed to have passed through two DLs.

tion, or for bias voltages larger than the plasma potential) the ion-beam energy returns to the same level as when the AP was biased at a negative potential.

\section{Aperture plate near center of the source chamber}

With the AP inserted into the plasma near the nozzle coil, the parallel ion kinetic energy at $z=3.0 \mathrm{~cm}$ increased from $9 \mathrm{eV}$ (Fig. 3) to $14 \mathrm{eV}$ (Fig. 4). To better understand the effect of the AP, we separated the AP (mechanical aperture) and nozzle (magnetic aperture) by positioning the AP near the center of the source (main) chamber, between -29.1 and $-29.4 \mathrm{~cm}$, see Fig. 2(d). The viewing geometry in this configuration allowed spatially resolved measurement of parallel ion flow speeds around both the mechanical and magnetic apertures. As shown in Fig. 7, ions begin to accelerate at $z=-31.4 \mathrm{~cm}$ and enter the aperture hole with an energy of $7.2 \mathrm{eV}$ at $z=-29.4 \mathrm{~cm}$ (Fig. 7). The ions keep accelerating as they transit the aperture and reach $20.4 \mathrm{eV}$ at $z=-28.9 \mathrm{~cm}$ (Fig. 7). Further downstream of the AP, at $z=-28.1 \mathrm{~cm}$, the ions reached $39.5 \mathrm{eV} \sim 7 k T_{e}$. Thus, the ions accelerated from 7.2 to $39.5 \mathrm{eV}$ in $1.2 \mathrm{~cm}, \sim 2000_{s} \lambda_{D}$ or $\sim 200_{\mathrm{ER}} \lambda_{D}$.

In the expansion region beyond the nozzle coil $(z>1 \mathrm{~cm})$, three ion populations are observed, see Fig. 7 . The LEP ions with parallel kinetic energy $\sim 0.1 \mathrm{eV}$ are produced locally in the expansion region. We suggest that the ions with kinetic energy $\sim 16.3 \mathrm{eV}$ at $z=4.4 \mathrm{~cm}(\sim 7 \mathrm{eV}$ at $z=3 \mathrm{~cm}$, as shown in Fig. 7) were created in the region between the AP and the nozzle coil and then accelerated through a DL at the nozzle, gaining $\sim 16 \mathrm{eV}$ in transit. A third, super-high-energy, population (SHEP) is observed downstream of the nozzle $(z=2.9 \mathrm{~cm})$ having a flow energy of $51 \mathrm{eV}$. The $51 \mathrm{eV}$ energy is consistent with the observation of a roughly $40 \mathrm{eV}$ energy increase at the AP followed by a $7-10 \mathrm{eV}$ increase at the magnetic nozzle at $z=2.9 \mathrm{~cm}$. In other words, this configuration of a mechanical AP followed by a magnetic nozzle leads to the formation of two distinct double layers.

Since a DL is essentially a plasma sheath that forms in the interior of a plasma, a presheath must arise to match the 


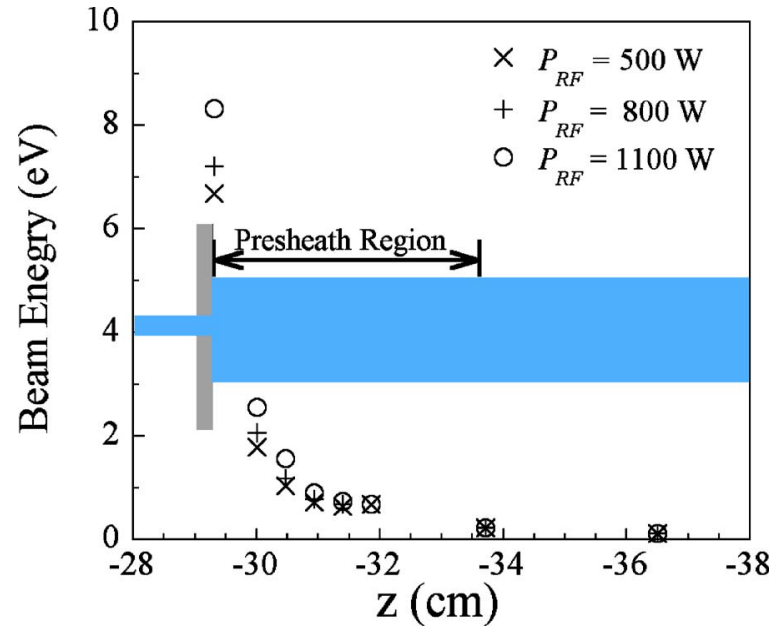

FIG. 8. The ion-beam energy in the presheath for $\mathrm{rf}$ powers of $500 \mathrm{~W}$ (solid circles), $800 \mathrm{~W}$ (solid squares), and $1100 \mathrm{~W}$ (solid diamonds). $B_{H}=580 \mathrm{G}$ and $P_{M}=0.5$ mTorr. Aperture plate at $z=-29.4 \mathrm{~cm}$ (right surface).

plasma potential to that of the DL. ${ }^{29}$ To satisfy the Bohm criterion for ions falling into the sheath at the edge of the DL, the ions must reach a minimum parallel energy of $\frac{1}{2} k T_{e}$ by passing through the presheath. The measured ion acceleration before the DL is shown in Fig. 8 for the AP placed at $z=-29.4 \mathrm{~cm}$. The ions begin to accelerate $\sim 3 \mathrm{~cm}$ before the plate, approximately equal to the expected length of the presheath, the ion-neutral collision length. ${ }^{21,22}$ The beam energies at the aperture are 6.7, 7.2, and $8.3 \mathrm{eV}$ for 500, 800, and $1100 \mathrm{~W}$ of $\mathrm{rf}$ power. Langmuir probe measurements at $z \approx-32 \pm 0.15 \mathrm{~cm}$ indicate that the electron temperatures are $8.0 \pm 1.0,8.4 \pm 1.0$, and $8.4 \pm 1.0 \mathrm{eV}$. Langmuir probe characteristics show an energetic electron population, if at all present, had a density less that $0.1 \%$ of the bulk electrons. Thus, the ion energies at the aperture indicate a $k T_{e} / e$ potential drop in transiting the presheath. The presheath region, as indicated in Fig. 8, is $4-5 \mathrm{~cm}$, which, as noted before, is approximately equal to the ion-neutral mean free path of $3-5 \mathrm{~cm}$. Thus, the thickness of the presheath is consistent with Riemann's sheath model. However, similar to Oksuz and Hershkowitz's experiment, ${ }^{22}$ the potential drop over the presheath is $\sim k T_{e} / e$, instead of $k T_{e} / 2 e$ in Riemann's model. The exiting ion flow energies at $z=-27.6 \mathrm{~cm}$, about $1.5 \mathrm{~cm}$ from the exit of aperture, are 36.5, 39.6, and $47.8 \mathrm{eV}$ for these three rf power scans, i.e., the strength of the sheath DL increases with increasing $\mathrm{rf}$ power.

Note that although the plasma parameters upstream of the nozzle are dramatically different in Figs. 4 and 7, the strength of the DLs formed by the nozzle magnetic field are nearly identical, about $20 \mathrm{~V}$ or $\sim 3 k T_{e} / e$. Although no spatial scan was performed for the configuration without an aperture plate, the increase in ion kinetic energy close to the magnetic aperture is approximately the same, $7.0 \mathrm{eV}$, at $z=3.0 \mathrm{~cm}$ with $B_{N}=2250 \mathrm{G}$, for configurations $2(\mathrm{a})$ and $2(\mathrm{~d})$. Thus, these measurements suggest that the nozzle magnetic field creates an overall $20 \mathrm{~V}$ potential drop along the axis even though the detailed DL structure does depend on the upstream plasma parameters (as indicated by Figs. 4 and 7).

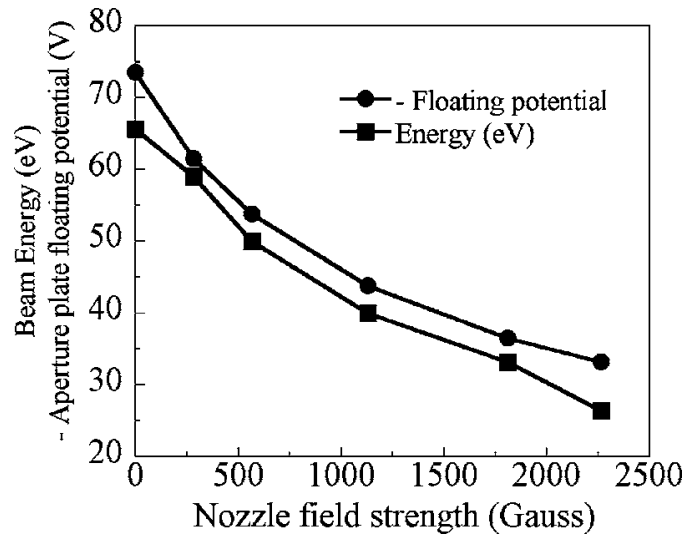

FIG. 9. The ion-beam energy (solid circles) at $z=14.8 \mathrm{~cm}$ and absolute value of aperture-plate floating potential (solid squares) vs the nozzle field strength for $\mathrm{rf}$ power of $720-850 \mathrm{~W}, B_{H}=580 \mathrm{G}, P_{M}=0.5 \mathrm{mTorr}$, and $P_{\mathrm{ER}}=0.12-0.24 \mathrm{~m}$ Torr. The aperture plate was at $z=14.0 \mathrm{~cm}$ (left surface) in the ER.

\section{Aperture plate in the expansion chamber}

The floating potential acquired by an electrically floating AP placed in the expansion region of the experiment is indicative of a density-weighted average energy of the electron population in the plasma. Shown in Fig. 9 are the measurements of the $z$-directed ion energy at $z=5.3 \mathrm{~cm}$ for the AP at $z=4.5 \mathrm{~cm}$ [the AP position as indicated by Fig. 2(e)] and the aperture-plate floating potential versus nozzle magnetic-field strength. Both the ion flow energy and the floating potential of the AP increase with decreasing nozzle magnetic-field strength. The large negative floating potential, up to $-75 \mathrm{~V}$, of the electrically isolated aperture plate in the expansion chamber suggests the existence of energetic electrons in the plasma. The existence of energetic electrons in helicon sources, possibly resulting from Landau damping of the helicon wave, has long been debated amongst the helicon source community. ${ }^{30}$ Reports of energetic electrons in long, lowaxial-power density, higher-neutral-pressure helicon plasmas indicated that the energetic population was less than $\sim 10^{-4}$ of the bulk, thus the Landau damping explanation for the high ionization efficiency of helicon sources has fallen into disfavor. $^{31,32}$ However, the LIF measurements presented here, for a relatively short, higher power-density device, indicate a strong correlation between the mechanism responsible for determining the strength of the DL and the floating potential of the AP-possibly a result of the DL formation being controlled by a population of energetic electrons in the helicon source.

If the high (negative) floating potential of the AP results from an energetic electron population, the same population of energetic electrons should determine the strength of the ion-accelerating DL and both the AP floating potential and the ion-beam energy will have similar dependencies on the source parameters. ${ }^{20}$ Note also that if the higher nozzle field strength results in more energetic electrons reflected back into the source because of magnetic mirroring, i.e., fewer energetic electrons can reach the AP downstream of nozzle, the decrease in the strength of the DL and the decrease in the AP floating potential with increasing nozzle magnetic field 
strength are easily explained. From a Laval-nozzle model, it is expected that an increasing magnetic-nozzle field strength should lead to higher-energy ion beams. In contrast, our results show that a weaker nozzle magnetic field does a poorer job of confining the energetic source electrons and is more effective at ion-beam acceleration. These results also imply that the energetic electrons have a significant perpendicular energy.

\section{SUMMARY}

Detailed measurements were made of the velocity distribution of free and trapped ions in the vicinity of single and multiple double-layer structures. Near and in the DL the trapped-ion velocity distribution is well represented by a single, nearly stationary Maxwellian velocity distribution. The measured free-ion speeds reveal that the DL formed by the nozzle is about $3 k T_{e} / e$, independent of the upstream IVDF and EEDF. Acceleration of ions up to-and exceeding - the ion sound speed (determined by the bulk electron temperature) is observed in the presheath upstream of the DL. The potential drop over the presheath is $\sim k T_{e} / e$.

Two-step double-layer structures were produced by first creating a DL at an electrically floating plate placed in the plasma-source chamber. Then, the plasma downstream of the first DL flowed through a second DL created by a rapid plasma expansion in the divergent magnetic field of a magnetic-nozzle coil. That a mechanical aperture can create a DL with strength $\sim 6 k T_{e} / e$ and thereby increase the exit velocity of ions flowing through an additional DL further downstream suggest that a sequence of appropriately sized apertures could be used to modify the specific impulse of plasma thrusters or other systems used to create ion beams.

Perhaps the most significant result from this work is that for expanding helicon source plasmas the ion beams created by the DL in a magnetic aperture appear to depend on the energetic electron population that can escape the source region. Therefore, as seen in other experiments, configurations with no nozzle magnetic field and very weak fields in the expansion region yield the highest-energy ion beams. Recent investigations by comparing different spectroscopic line ratios also suggest the existence of a small population $(\sim 0.1 \%)$ of suprathermal electrons $\left(\sim 10 k T_{e}\right) .^{33}$

Further studies are still needed to explore the relationship among the DL strength and aperture size, the dependence of the threshold pressure for DL formation on gas species and neutral-gas temperature, and the effects of multiple gas species on the strength of the DL.

\section{ACKNOWLEDGMENTS}

We thank Bruce Berlinger for excellent technical support.

This work was supported, in part, by U.S. Department of Energy Contract No. DE-AC02-76-CHO-3073 and NSF Grant No. PHY-0315356.

${ }^{1}$ M. A. Raadu, Phys. Rep. 178, 25 (1989), and references therein.

${ }^{2}$ L. R. Block, Astrophys. Space Sci. 55, 59 (1978).

${ }^{3}$ B. H. Quon and A. Y. Wong, Phys. Rev. Lett. 37, 1393 (1976).

${ }^{4}$ E. E. Scime, P. A. Keiter, S. P. Gary, M. Balkey, R. F. Boivin, J. L. Kline, and M. Blackburn, Phys. Plasmas 7, 2157 (2000).

${ }^{5}$ X. Sun, C. Biloiu, R. Hardin, and E. E. Scime, Plasma Sources Sci. Technol. 13, 359 (2004).

${ }^{6}$ S. A. Cohen, N. S. Siefert, S. Stange, R. F. Boivin, E. E. Scime, and F. M. Levinton, Phys. Plasmas 7, 2593 (2003).

${ }^{7}$ C. Charles and R. W. Boswell, Appl. Phys. Lett. 82, 1356 (2003).

${ }^{8}$ C. Charles, A. W. Degeling, T. E. Sheridan, J. H. Harris, M. A. Lieberman, and R. W. Boswell, Phys. Plasmas 7, 5232 (2000).

${ }^{9}$ C. Charles, Appl. Phys. Lett. 84, 332 (2004).

${ }^{10}$ R. F. Boivin and E. E. Scime, Rev. Sci. Instrum. 74, 4352 (2003).

${ }^{11}$ P. C. Stangeby, Plasma Sheath in Physics of Plasma-Wall Interactions in Controlled Fusion Devices (Plenum, New York, 1984).

${ }^{12}$ S. A. Andersen, V. O. Jensen, P. Nielsen, and N. D' Angelo, Phys. Fluids 12, 557 (1969).

${ }^{13}$ A. V. Arefiev and B. N. Breizman, Phys. Plasmas 11, 2942 (2004).

${ }^{14}$ F. Chang-Diaz, Sci. Am. 283, 90 (2002).

${ }^{15}$ F. W. Perkins and Y. C. Sun, Phys. Rev. Lett. 46, 115 (1981).

${ }^{16}$ R. Schrittwieser, I. Axnas, T. Carpenter, and S. Torven, IEEE Trans. Plasma Sci. 20, 607 (1992)

${ }^{17}$ A. Meige, R. W. Boswell, C. Charles, and M. M. Tuner, Phys. Plasmas 12, 052317 (2005).

${ }^{18}$ N. Plihon, C. S. Corr, and P. Chabert, Appl. Phys. Lett. 86, 091501 (2005).

${ }^{19}$ K. Sato and F. Miyawaki, Phys. Fluids B 4, 1247 (1992).

${ }^{20}$ G. Hairapetian and R. Stenzel, Phys. Fluids B 3, 899 (1991).

${ }^{21}$ K. U. Riemann, Phys. Plasmas 4, 4158 (1997).

${ }^{22}$ L. Oksuz and N. Hershkowitz, Phys. Rev. Lett. 89, 145001 (2002).

${ }^{23}$ R. W. Boswell, A. J. Lichtenberg, and D. Vender, IEEE Trans. Plasma Sci. 20, 62 (1992).

${ }^{24}$ K. Shiraishi and S. Takamura, J. Nucl. Mater. 176-177, 251 (1990).

${ }^{25}$ M. Cercek and T. Gyergyek, J. Phys. D 34, 330 (2001).

${ }^{26}$ X. Sun, A. M. Keesee, C. Biloiu, E. E. Scime, A. Meige, C. Charles, and R. W. Boswell, Phys. Rev. Lett. 95, 025004 (2005).

${ }^{27}$ N. Hershkowitz, Space Sci. Rev. 41, 351 (1985).

${ }^{28}$ G. Hairapetian and R. Stenzel, Phys. Rev. Lett. 65, 175 (1990).

${ }^{29}$ N. Hershkowitz, IEEE Trans. Plasma Sci. 22, 11 (1994).

${ }^{30}$ F. F. Chen, Plasma Phys. Controlled Fusion 33, 339 (1991).

${ }^{31}$ A. W. Molvik, T. D. Rognlien, J. A. Byers, R. H. Cohen, A. R. Ellingboe, E. B. Hooper, H. S. McLean, B. W. Stallard, and P. A. Vitello, J. Vac. Sci. Technol. A 14, 984 (1996).

${ }^{32}$ F. F. Chen and D. D. Blackwell, Phys. Rev. Lett. 82, 2677 (1999).

${ }^{33}$ S. A. Cohen, X. Sun, N. M. Ferraro, E. E. Scime, M. Miah, S. Stange, N. Siefert, and R. Boivin, IEEE Trans. Plasma Sci. (submitted). 